\title{
Analytical Electron Microscopy Studies of the CMSX-4 Single Crystal Superalloy Subjected to High Temperature Annealing
}

\author{
B. Dubiel ${ }^{a, *}$, P. Indyka ${ }^{b}$, I. KAlEmba-ReC ${ }^{a}$ And T. MoskalewiCZ ${ }^{a}$ \\ ${ }^{a}$ AGH University of Science and Technology, Faculty of Metals Engineering and Industrial Computer Science, \\ Al. A. Mickiewicza 30, 30-059 Kraków, Poland \\ ${ }^{b}$ Jagiellonian University, Faculty of Chemistry, R. Ingardena 3, 30-060 Kraków, Poland
}

\begin{abstract}
The aim of the present work is to investigate the development of the $\gamma / \gamma^{\prime}$ microstructure and the changes in chemical composition of $\gamma$ and $\gamma^{\prime}$ phases during high temperature annealing of CMSX-4 superalloy at a temperature of $1100^{\circ} \mathrm{C}$ in the time range from 500 to $2500 \mathrm{~h}$. The studies were performed by means of scanning electron microscopy and the modern scanning-transmission electron microscopy with Super-X EDS system of four SDD detectors. Results of scanning electron microscopy and scanning-transmission electron microscopy analyses have shown that the microstructure of CMSX-4 superalloy is unstable during ageing at high temperature and the coalescence of cuboidal $\gamma^{\prime}$ precipitates was observed. Energy dispersive X-ray microanalysis revealed the distribution of particular alloying elements in the $\gamma$ and $\gamma^{\prime}$ phases and the differences in their concentration in the function of the annealing time.
\end{abstract}

DOI: 10.12693/APhysPolA.131.1375

PACS/topics: 61.66.Dk, 68.37.Hk, 68.37.Lp, 68.37.Ma, 82.80.Ej

\section{Introduction}

CMSX-4 is a second generation single crystal (SC) nickel-base superalloy applied for gas turbine blades. The name of the CMSX superalloy grades is the acronym of the Cannon-Muskegon single crystal [1]. Chemical composition of CMSX-4 was developed to obtain optimized microstructure and low level of residual microsegregation [2]. Its microstructure consists of intermetallic $\gamma^{\prime}$ phase cuboidal precipitates embedded in narrow channels of $\gamma$ solid solution matrix. Between those two phases the negative misfit of the lattice parameters was measured by X-ray diffraction [3]. During prolonged exposure at high temperature the coagulation of $\gamma^{\prime}$ phase occurs. The evolution of the $\gamma^{\prime}$ phase in SC nickel-base superalloys was the subject of many studies [4-12]. Especially, the directional coagulation under the influence of external stress, generally known as rafting, was studied in detail in many superalloys [4-8]. However, reports on the $\gamma^{\prime}$ evolution during annealing without the external load are very limited [9-12]. The preferentially-oriented plates of the $\gamma^{\prime}$ phase formed at high temperature without applied stress was observed first by Hazotte and Lacaze [9]. They postulated that the inhomogeneity of chemical composition related with the dendritic structure can generate the chemical gradients, which influence the preferred direction of the $\gamma^{\prime}$ plates formed during coarsening. Epishin et al. [10] have proposed that the segregation of chemical elements during solidification would effect in thermal contraction during precipitation of $\gamma^{\prime}$ phase and thus generate internal dendritic stresses. Reed et al. [8] showed

*corresponding author; e-mail: bdubiel@agh.edu.pl that residual microsegregation in the dendritic and interdendritic regions in CMSX-4 superalloy results in different rates of $\gamma^{\prime}$ coarsening in those areas. The mechanism of $\gamma^{\prime}$ rafting without applied stress in the single crystal superalloy CMSX-4 was investigated also by Cheng et al. [11]. They have observed the formation of $\gamma^{\prime}$ rafts preferentially oriented in the directions perpendicular to the dendrite arms and suggested that it is influenced by the internal chemical gradients. The above consideration was mostly focused on the phenomena occurring at the microscale. To investigate in more detail the coarsening of the $\gamma^{\prime}$ phase, the evolution of the dislocation substructure and the diffusion of the chemical elements between $\gamma$ and $\gamma^{\prime}$ phases should be examined. Although the dislocation analysis attained the high interest, the influence of chemical composition of $\gamma$ and $\gamma^{\prime}$ phases on the development of rafts is not fully understood, mainly due to the limits of experimental methods used for microanalysis in nanoareas. Nowadays it can be thoroughly investigated with use of the most advanced analytical electron microscopy methods. Recently, the advances in analytical electron microscopy enabled to perform both microstructural analysis and microanalysis of chemical composition with high spatial resolution at the nanoscale.

In the present work to investigate the development of the $\gamma / \gamma^{\prime}$ microstructure and the changes in chemical composition of $\gamma$ and $\gamma^{\prime}$ phases during high temperature annealing of CMSX-4 superalloy we have used scanning electron microscopy (SEM) and scanning-transmission electron microscopy (STEM) combined with advanced energy dispersive X-ray microanalysis (EDS). The combination of the modern analytical electron microscopy methods contributed to better understanding of the $\gamma^{\prime}$ phase coarsening in CMSX-4 SC superalloy. 


\section{Materials and experimental}

The $\langle 001\rangle$ oriented bars of CMSX-4 single crystal superalloy subjected to standard heat treatment were delivered by Howmet Ltd, UK. The alloy contains 10 major alloying elements and its chemical composition is following (in wt\%): $\mathrm{Ni}-9.5 \mathrm{Co}-6.4 \mathrm{Cr}-6.4 \mathrm{Ta}-6.4 \mathrm{~W}-$ $5.6 \mathrm{Al}-2.9 \mathrm{Re}-1.0 \mathrm{Ti}-0.6 \mathrm{Mo}-0.1 \mathrm{Hf}$. To investigate the microstructural changes caused by the high temperature exposure, the specimens cut perpendicular to [001] crystallographic direction in the single crystal were isothermally annealed at a temperature of $1100{ }^{\circ} \mathrm{C}$ in the time range from 500 to $2500 \mathrm{~h}$ and subsequently cooled in air. SEM investigation was performed using an FEI Nova NanoSEM 450 microscope equipped with an EDS spectrometer of EDAX. STEM investigation has been carried out in high angle annular dark-field (HAADF) mode by means of FEI Tecnai Osiris microscope equipped with X-FEG high brightness electron source and Super$\mathrm{X}$ EDS system of four SDD detectors. EDS maps were acquired in STEM mode with the resolution $1024 \times 1024$ pixels and the pixel size of $2 \mathrm{~nm}$. For acquisition of EDS maps the $K_{\alpha}$ peaks of $\mathrm{Ni}, \mathrm{Co}, \mathrm{Cr}, \mathrm{Ti}, \mathrm{Al}, \mathrm{Mo}$ and $L_{\alpha}$ peaks of $\mathrm{Ta}, \mathrm{Re}, \mathrm{W}$ were used. The $\mathrm{Cu} K_{\alpha}$ peak of specimen holder was deconvoluted. The EDS data were stored for every point in the scanned area. Quantitative EDS maps and line profiles were reconstructed using ESPRIT software of Bruker with the averaging of 5 pixels, which corresponds to $10 \mathrm{~nm} \times 10 \mathrm{~nm}^{2}$ square units. Based on the results of quantitative EDS analysis the average chemical composition of $\gamma$ and $\gamma^{\prime}$ phases was determined.

\section{Results and discussion}

The SEM investigation enabled to examine the $\gamma / \gamma^{\prime}$ microstructure development during prolonged annealing at temperature of $1100{ }^{\circ} \mathrm{C}$ (Fig. $\left.1 \mathrm{~A}-\mathrm{C}\right)$. It was observed that the $\gamma$ phase channels were gradually dissolved and the coalescence of $\gamma^{\prime}$ precipitates occurred. As the result, the $\gamma^{\prime}$ phase becomes the matrix containing discontinuous $\gamma$ phase. Beside the coalescence of the $\gamma^{\prime}$ phase, the formation of pores and precipitation of the topologically close packed (TCP) phases occurred, as can be seen in Fig. 1B,C. The microstructure and chemical composition of TCP phases was the subject of our previous work [12]. We have demonstrated the compositional variations of $\gamma$, $\gamma^{\prime}$ and TCP phases for the as-received condition and annealed at $1100^{\circ} \mathrm{C}$ for $2500 \mathrm{~h}$. In this work we apply the same method of EDS microanalysis in STEM for determination of elemental compositions of $\gamma$ and $\gamma^{\prime}$ phases as a function of annealing time at $1100^{\circ} \mathrm{C}$.

A STEM-HAADF image of the specimen annealed at a temperature of $1100^{\circ} \mathrm{C}$ for $500 \mathrm{~h}$, together with the corresponding EDS maps, is shown in Fig. 2. The contrast in STEM-HAADF images depends on the intensity of the thermal diffuse scattered (TDS) electrons, thus the image intensity depends on the atomic number $Z$ and the specimen thickness [13]. Furthermore, the local atomic rearrangements, which occur around dislocations, also give
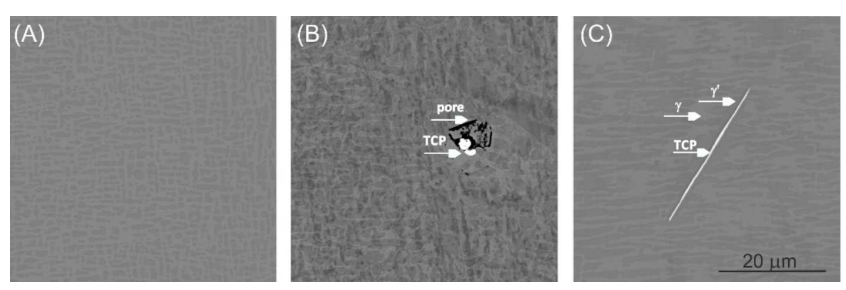

Fig. 1. SEM images of the CMSX-4 microstructure after annealing at temperature of $1100{ }^{\circ} \mathrm{C}$ for (A) $500 \mathrm{~h}$, (B) $1000 \mathrm{~h}$, and (C) $2500 \mathrm{~h}$.

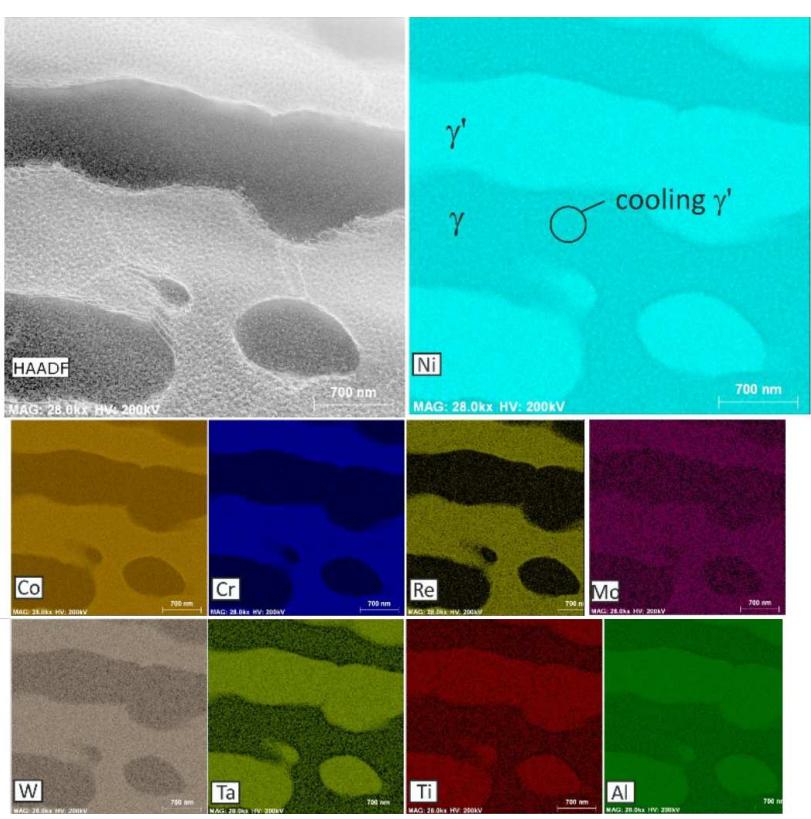

Fig. 2. STEM-HAADF image of the microstructure of CMSX-4 superalloy annealed at temperature of $1100^{\circ} \mathrm{C}$ for $500 \mathrm{~h}$ and EDS elemental maps of chemical elements distribution in $\gamma$ and $\gamma^{\prime}$ phases.

rise to diffuse scattering, similar to TDS [14] and thus the dislocations occur as the bright lines. As can be seen in STEM-HAADF image in Fig. 2, $\gamma$ phase containing chemical elements with higher mean atomic number exhibits brighter contrast than $\gamma^{\prime}$ phase, containing lighter elements, so the image intensity is mainly modulated by $Z$-contrast. The bright contrast of dislocations accumulated at the $\gamma / \gamma^{\prime}$ interfaces can also be observed.

The formation of the interface dislocations has been reported to be related with the relaxation of $\gamma / \gamma^{\prime}$ coherency stresses [15]. Relieve of the lattice misfit can influence the change of chemical potential of atoms and thus provide the easy diffusion paths, enabling dissolution of $\gamma$ channels and coalescence of $\gamma^{\prime}$ cubes.

EDS measurements were performed in the areas located the far from the TCP phases. The EDS maps revealed the partitioning of $\mathrm{Al}, \mathrm{Ti}$, Ta, and $\mathrm{Ni}$ to the $\gamma^{\prime}$ phase as well as partitioning of $\mathrm{Co}, \mathrm{Cr}, \mathrm{Re}, \mathrm{Mo}$, and $\mathrm{W}$ to the $\gamma$ phase (Fig. 2). The resolution of the EDS 
maps was high enough to observe the fine nanoparticles containing $\gamma^{\prime}$-forming elements inside $\gamma$ phase channels, visible in magnified $\mathrm{Ni}$ map in Fig. 2. In the literature the small $\gamma^{\prime}$ particles occurring in the Ni-base superalloys are termed cooling $\gamma^{\prime}$ or tertiary $\gamma^{\prime}$ [16]. Their nucleation is associated with the diffusion of $\gamma^{\prime}$ stabilising elements inside the $\gamma$ phase channels during cooling from the annealing temperature. In our experiments the specimens were cooled in air, but the cooling rate was not fast enough to prevent precipitation processes.
Although EDS measurements were performed with high spatial resolution, it was difficult to perform quantitative analysis of cooling $\gamma^{\prime}$ nanoparticles composition, because they are embedded in the specimens about $100 \mathrm{~nm}$ thick. Therefore it was assumed that the averaged chemical composition in the areas of $\gamma$ channels and $\gamma^{\prime}$ agglomerates corresponds to the composition of the $\gamma$ and $\gamma^{\prime}$ phases at the annealing temperature. For simplicity these areas are called $\gamma$ and $\gamma^{\prime}$.
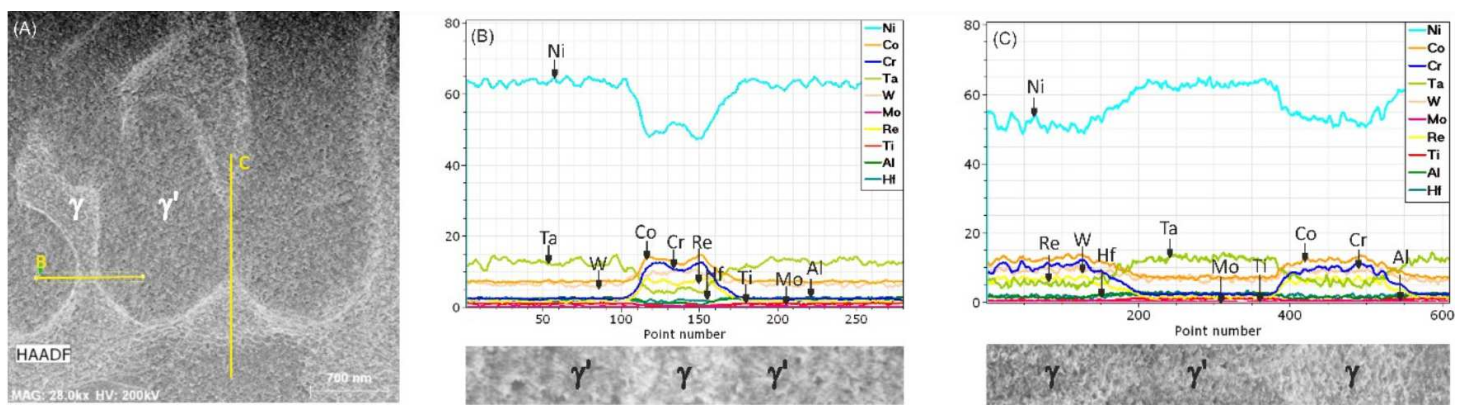

Fig. 3. STEM-HAADF image (A) and the quantitative EDS line profiles (B), (C) along the lines marked by B and C in (A) in specimen annealed at temperature of $1100^{\circ} \mathrm{C}$ for $2500 \mathrm{~h}$.

To determine the concentration changes in the function of the annealing time, quantitative EDS elemental profiles were created across $\gamma$ and $\gamma^{\prime}$ in the submicron areas. Figure 3 shows STEM-HAADF image of the microstructure of the specimen annealed at temperature of $1100{ }^{\circ} \mathrm{C}$ for $2500 \mathrm{~h}$ (Fig. 3A) and the example line scans acquired across lines marked by $\mathrm{B}$ and $\mathrm{C}$ (Fig. 3B,C).

The STEM-HAADF image in Fig. 3A shows the differences in the intensity of the $\gamma$ and $\gamma^{\prime}$ areas, which are related with the $Z$-contrast, as well as the dislocations at the $\gamma-\gamma^{\prime}$ boundaries, appearing as bright lines. The line scans in Fig. 3B and $\mathrm{C}$ confirmed the qualitative results obtained by elemental maps and indicated the increased concentration of $\mathrm{Ni}$ and $\mathrm{Ta}$ in $\gamma^{\prime}$ phase as well as segregation of $\mathrm{Co}, \mathrm{Cr}, \mathrm{Re}$, and $\mathrm{W}$ to the $\gamma$ phase. The profiles shown in Fig. 3B were recorded across the relatively thick $\gamma$ channel. The slight increase of $\mathrm{Cr}$ and Co concentration, associated with depletion in $\mathrm{Ni}$ was observed at the $\gamma / \gamma^{\prime}$ interfaces. It cannot be excluded that such increase is not related with the inclination of the interfaces along the specimen thickness. The EDS line scan acquired in the area where the morphology of narrowed $\gamma$ channel indicated that it was dissoluted, revealed different shape of the concentration profiles (Fig. 3C). The smooth slope of the Co, Cr, Ni, and Ta profiles at the $\gamma / \gamma^{\prime}$ interfaces indicated the lack of segregation at the nanoscale associating the coalescence of $\gamma^{\prime}$ particles.

The averaged mass concentrations of particular chemical elements in $\gamma$ and $\gamma^{\prime}$ areas were calculated from the EDS data stored in every pixel of line scans and visualised in the form of plots in Fig. 4. The data determined for as-received condition are also given. The error bars represent the standard deviation of the measurements. It has to be mentioned that the sum concentrations of par-

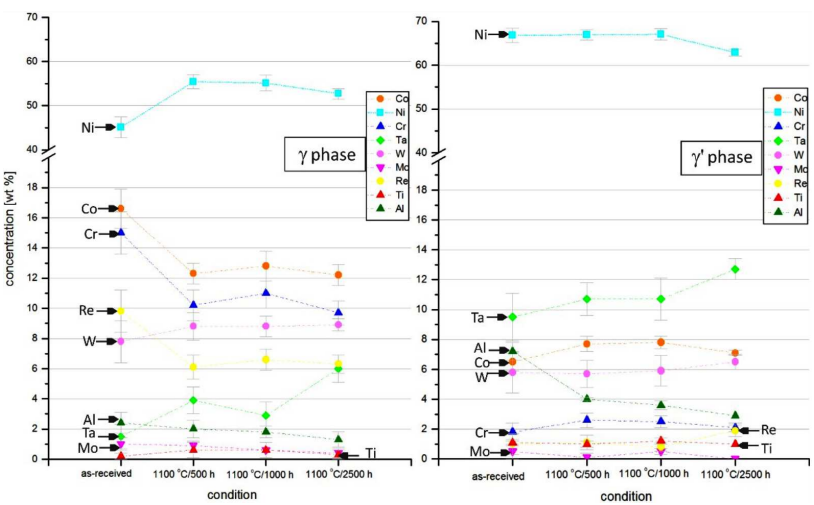

Fig. 4. The plots of the averaged concentration of chemical elements in $\gamma$ and $\gamma^{\prime}$ phases in the as-received condition and after annealing at temperature of $1100^{\circ} \mathrm{C}$ for 500,1000 , and $2500 \mathrm{~h}$.

ticular elements are not equal to $100 \%$ because they have been not weighted by the volume fractions of $\gamma$ and $\gamma^{\prime}$ phases, which are varied with temperature and time of high temperature annealing. Moreover, the changes in the composition of $\gamma$ and $\gamma^{\prime}$ phases are also influenced with the precipitation and growth of TCP phases, which in the specimen annealed at temperature of $1100^{\circ} \mathrm{C}$ for $2500 \mathrm{~h}$ contain $35.9 \mathrm{Re}, 30.9 \mathrm{~W}, 10.3 \mathrm{Ni}, 7.1 \mathrm{Co}, 6.9 \mathrm{Cr}$, $6.3 \mathrm{Ta}$, and $0.4 \mathrm{Mo}$ (in wt\%) [12].

The plots in Fig. 4 show that the concentration of $\mathrm{Co}, \mathrm{Cr}$, and Re in $\gamma$ phase decreases with a function of annealing time. Due to the dissolution of the $\gamma$ channels, the atoms of these $\gamma$-forming elements are embedded mainly in the TCP phases and in minor amount to the coarsened $\gamma^{\prime}$ agglomerates. The concentration of $\mathrm{W}$, 
which is also $\gamma$-forming element, remains at the similar level. The observed concentration changes of strengthening elements in $\gamma$ phase are expected to influence the decrease of the solid solution hardening. Although Ta is $\gamma^{\prime}$-forming element, in the present study the increase of its concentration in $\gamma$ phase was observed with prolongation of the annealing time. Additionally, in our previous work the contribution of Ta to the formation of TCP phases in CMSX-4 was revealed [12]. Therefore it may be suspected that the diffusion of Ta also plays an important role in the microstructural instability of CMSX-4 superalloy at high temperature. The most remarkable differences in $\gamma^{\prime}$ phase composition concern the increase in concentration of Ta, Co, and $\mathrm{Cr}$, which segregate to $\gamma^{\prime}$ phase from dissolved $\gamma$ phase. The pronounced decrease in $\mathrm{Al}$ content in $\gamma^{\prime}$ phase can be related with formation of $\mathrm{Al}_{2} \mathrm{O}_{3}$ scale.

\section{Conclusions}

The results of our measurements performed by means of high spatial resolution EDS microanalysis in STEM show the remarkable concentration changes in the chemical elements distribution in $\gamma$ and $\gamma^{\prime}$ phases during annealing at high temperature of $1100^{\circ} \mathrm{C}$. Based on the achieved results, the following conclusions can be drawn:

1. STEM-HAADF imaging revealed that the coalescence of the $\gamma^{\prime}$ particles during high temperature annealing is associated with the accumulation of dislocations at the $\gamma / \gamma^{\prime}$ interfaces. It suggests that such dislocation substructure can relieve the coherency stresses and facilitate the diffusion of elements between $\gamma$ and $\gamma^{\prime}$ phases.

2. The EDS mapping confirmed the segregation of Co, $\mathrm{Cr}, \mathrm{Re}, \mathrm{Mo}$, and $\mathrm{W}$ to the $\gamma$ phase and depletion of $\mathrm{Ni}, \mathrm{Al}, \mathrm{Ti}$, and Ta. The high spatial resolution of EDS maps enabled to detect nanoparticles rich in $\gamma^{\prime}$-forming elements, precipitated within $\gamma$ phase. Such nanoparticles are tertiary $\gamma^{\prime}$, precipitated during cooling from the annealing temperature.

3. Comparison of the quantitative EDS concentration profiles across the stable and dissolving $\gamma$ channels indicates the slight enrichment in $\mathrm{Co}$ and $\mathrm{Cr}$ at the stable interfaces and the broad concentration gradients at the interfaces moved due to the coalescence of $\gamma^{\prime}$ particles. This may suggest that the $\mathrm{Cr}$ and Co segregation can inhibit the diffusive movement of the $\gamma / \gamma^{\prime}$ interfaces.

4. Results of quantitative EDS microanalysis averaged over $\gamma$ phase nanoareas indicated the reduction in concentration of $\mathrm{Co}, \mathrm{Cr}$, and Re and an increase in Ta with prolongation of the high temperature annealing. The observed enrichment in Ta, which is $\gamma^{\prime}$-forming element, suggests that at high temperature the diffusion of Ta to the $\gamma$ phase may be favourable.
Consistently, a distinctive increase in Ta, Co, and Cr concentration in the $\gamma^{\prime}$ phase was detected. In addition, the reduction in $\mathrm{Al}$ content in $\gamma^{\prime}$ phase was found, which can be associated with the high temperature oxidation and formation of $\mathrm{Al}_{2} \mathrm{O}_{3}$ scale.

\section{Acknowledgments}

This work was supported by the Polish National Science Centre, project no. 2012/07/B/ST8/03392. The authors thank Howmet Exeter, UK for providing the CMSX-4 material. We also thank Dr. Marta Gajewska and M.Sc. Wiesław Brzegowy from AGH-UST for the specimen preparation.

\section{References}

[1] K. Harris, G.L. Erickson, R.E. Schwer, in: JOM J. Min. Met. Mat. S 35, 53 (1983).

[2] K. Harris, G.L. Erickson, S.L. Sikkenga, W.D. Brentnall, J.M. Aurrecoechea, S.L. Sikkenga, K.G. Kubarych, in: Superalloys 1992, Eds. S.D. Antolovich, R.W. Stusrud, R.A. MacKay, D.L. Anton, T. Khan, R.D. Kissinger, D.L. Klarstrom, TMS, Warrendale (PA) 1992, p. 297.

[3] B. Dubiel, Microstructural Changes during Creep of Single-Crystalline Nickel-Base Superalloys, AGH University of Science and Technology Press, Kraków 2011.

[4] J.K. Tien, S.M. Copley, Metall. Trans. 2, 215 (1971).

[5] T.M. Pollock, A.S. Argon, Acta Metall. Mater. 42, 1859 (1994).

[6] F.R.N. Nabarro, Met. Mater. Trans. A 27, 513 (1996).

[7] M. Veron, Y. Brechet, F. Louchet, Scr. Metall. 34, 1883 (1996).

[8] R.C. Reed, D.C. Cox, C.M.F. Rae, Mater. Sci. Tech. 23, 893 (2007).

[9] A. Hazotte, J. Lacaze, Scr. Metall. 23, 1877 (1989).

[10] A. Epishin, T. Link, U. Brückner, B. Fedelich, P.D. Portella, in: Superalloys 2004, Eds. K. Green, T.M. Pollock, H. Harada, T.E. Howson, R.C. Reed, J.J. Schirra, S. Walston, TMS, Warrendale (PA) 2004, p. 537.

[11] K.Y. Cheng, C.Y. Jo, D.H. Kim, T. Jin, Z.Q. Hua, Mater. Character. 60, 210 (2009).

[12] B. Dubiel, P. Indyka, I. Kalemba-Rec, T. Moskalewicz, Acta Phys. Pol. A 130, 1110 (2016).

[13] M. Shiojiri, H. Saijo, J. Microsc. 223, 172 (2006).

[14] S.J. Pennycook, P.D. Nellist, in: Impact of Electron and Scanning Probe Microscopy on Materials Research, Eds. D.G. Rickerby, G. Valdré, U. Valdré, Kluwer Academic, 1999, p. 161.

[15] J.Y. Buffiere, M. Ignat, Acta Metall. Mater. 43, 1791 (1995).

[16] R.C Reed, The Superalloys. Fundamentals and Applications, Cambridge University Press, 2006. 